\title{
Calidad de vida relacionada con la salud en adultos con normopeso, sobrepeso y obesidad
}

\section{Health-related quality of life in normal weight, overweight and obese adults}

Giovanny Arzate Hernández', Rosalinda Guadarrama Guadarrama ${ }^{1,2}$, Marcela Veytia López ${ }^{1,2}$, Rebeca María Elena Guzmán Saldaña ${ }^{3}$

${ }^{1}$ Facultad de Ciencias de la Conducta de la Universidad Autónoma del Estado de México

${ }^{2}$ Instituto de Estudios Sobre la Universidad de la Universidad Autónoma del estado de México

${ }^{3}$ Instituto de Ciencias de la Salud de la Universidad Autónoma del Estado de Hidalgo

Recibido: 02 de agosto de 2018

Revisado: 21 de septiembre de 2018

Aceptado: 05 de diciembre de 2018

Autora de correspondencia: rossygma@hotmail.com (R. Guadarrama-Guadarrama)

Financiamiento: Ninguno

Agradecimientos

Conflicto de intereses: Los autores declaran no tener conflicto de intereses.

Resumen Actualmente el sobrepeso y la obesidad presentan una alta prevalencia a nivel mundial, y se encuentran relacionados con enfermedades crónico-degenerativas, mortalidad prematura y disminución en la calidad de vida. El propósito de esta investigación fue comparar la calidad de vida relacionada con la salud (CVRS) en función del estado de nutrición (EN) y sexo. Participaron 202 adultos ( 84 hombres y 118 mujeres), con una edad entre 18 y 81 años $(M=39.74 D E=13.77)$, quienes fueron distribuidos por su EN en: normopeso, sobrepeso y obesidad. Los participantes completaron el cuestionario 36-Item Short Form Health Survey (SF-36). En la muestra predominó la dimensión Función física (FF), considerándola muy buena. Las dimensiones Rol emocional (RE), Rol físico (RF), Vitalidad (VT) y Salud mental (SM) se ubicaron en el nivel de bueno, y solamente la Salud general (SG) se consideró regular. En el caso de la comparación entre los grupos en función del EN no se encontraron diferencias significativas; sin embargo, en la comparación por sexo, los hombres obtuvieron mayores puntuaciones 
en: FF, SM, VT y RE. Se concluye que no hay diferencia estadísticamente significativa de la CVRS entre los grupos por EN, no obstante, al realizar la comparación por sexo se encuentran diferencias significativas a favor de los hombres.

Palabras clave. Calidad de vida, Adultos, Normopeso, Sobrepeso, Obesidad.

\begin{abstract}
Overweight and obesity currently have a high prevalence worldwide, are related to chronic degenerative diseases, premature mortality and the decline in quality of life. The purpose of the present investigation was to compare the quality of life related to health (HRQOL) between groups by their nutritional status (NS) and by sex. There was a sample of 202 participants ( 84 men and 118 women) with an age between 18 and 81 years $(M=39.74 S D=13.77)$, distributed by their NS in: normal weight, overweight and obesity, they were administered the questionnaire 36-Item Short Form Health Survey (SF-36). In the general sample, the Physical Function (PF) dimension was predominant, being considered as very good, the scores obtained in the Emotional Role (ER), Physical Role (PR), Vitality (VT) and Mental Health (MH) dimensions were in a range estimated as good and only the General Health (GH) dimension was considered regular. In the case of the comparison between the groups by state of nutrition, no statistically significant differences were found between them, however, in the comparison made by sex, statistically significant differences were found in the factors of PF, MH, VT and ER in favor of the group of men. In conclusion, there is not statistically significant difference of the HRQoL between the groups by NS, nevertheless, when performing the comparison by sex, significant differences are found in favor of the men.
\end{abstract}

Keywords. Quality of life, Adults, Normal weight, Overweight, Obesity.

\section{Introducción}

A nivel mundial la prevalencia del sobrepeso y la obesidad ha tenido aumento en los últimos años, motivo por el cual la Organización Mundial de la Salud (OMS) ha declarado un estado de "epidemia global de la obesidad” (Barrera-Cruz, Molina-Ayala y Rodríguez-González, 2013; López-Alarcón y Rodríguez-Cruz, 2008), considerando la elevada tasa como un problema de salud pública (Alcaraz, Ferrer y $\mathrm{Pa}$ rrón, 2015; Gálvez et al., 2015; Salazar, Torres, Aranda y López, 2016). Tanto el sobrepeso como la obesidad se caracterizan por el aumento de grasa corporal (Moreno, 2012), con una etiología de tipo multifactorial, puesto que son el resultado de diversas dimensiones, como son: culturales, ambientales, metabólicas y genéticas (Barquera-Cervera, Campos-Nonato, Rojas y Rivera, 2010; Jiménez, Suárez y Díaz, 2013).
Anteriormente, tanto el sobrepeso como la obesidad eran considerados un problema exclusivo de países con ingresos altos (Alcaraz et al., 2015), sin embargo, actualmente estas enfermedades tienen gran prevalencia en aquellos países que se encuentran en vías de desarrollo (Bertheau, Cruz-Quintana, Pappous y Rio-Valle, 2016; García-García et al., 2008).

En México, al igual que otros países que se encuentran en vías de desarrollo, el perfil epidemiológico ha venido presentados cambios, en los cuales, tanto las deficiencias nutricionales, la obesidad y las enfermedades que derivan de estas coexisten entre sí (López-Alarcón y Rodríguez-Cruz, 2008). En el 2006 se reportó que la prevalencia de sobrepeso y obesidad en adultos mayores de 20 años fue de $69.7 \%$, ubicándolo como uno de los países con mayores casos dentro de América y a nivel global; posteriormente, en el 2012 dichas enfermedades aumentaron, reportando que 
71.3\% delos adultos mexicanos padecen sobrepeso-con mayor prevalencia en mujeres-y obesidad-con mayor prevalencia en hombres- (Barquera, Campos-Nonato, Hernández-Barrera, Pedroza y Rivera-Dommarco, 2013; Esquivias-Zavala, Reséndiz-Barragán, García y Elías-López, 2016); sin embargo, en el Estado de México el incremento de la obesidad se presenta en todos los grupos poblacionales, con un $47.7 \%$ en hombres y $42.6 \%$ en mujeres (Salazar et al., 2016). Inclusive, estudios realizados en Estados Unidos indican que las tasas de sobrepeso y obesidad son mayores en los México-Americanos en comparación con la población caucásica (López-Alarcón y Rodríguez-Cruz, 2008).

$\mathrm{El}$ aumento descontrolado en los últimos 30 años está asociado a cambios en los hábitos alimentarios, destacando el consumo de alimentos energéticamente densos (Jiménez et al., 2013). Actualmente la obesidad se ha convertido en foco de interés por diversas disciplinas, sin embargo, a pesar de que en México el desarrollo de las investigaciones básicas y clínicas de la obesidad son numerosas, la difusión de los resultados y la comunicación entre profesionales no es suficiente (García-García et al., 2008).

La obesidad, al igual que otras enfermedades como las de tipo infecciosas, cáncer y enfermedades mentales, es una enfermedad progresiva; no obstante, se puede revertir y controlar en su fase inicial. Pese a que existen aproximadamente 18 millones de obesos en México, en los centros de salud sólo se atiende a aquellos que buscan atención médica (García-García et al., 2008). Al ser visualizada como una epidemia mundial, se debe hacer hincapié en todas aquellas consecuencias que conlleva (Esquivias-Zavala et al., 2016), como son las enfermedades crónico-degenerativas, además de mayor mortalidad prematura y disminución en la calidad de vida (Barrera-Cruz et al., 2013), la cual es considerada como un constructo de tipo multifactorial, en el cual se ven incluidos aspectos asociados tanto a la salud física como mental (Dueñas, Del Carmen, Zamora y Saunas, 2005; Miró, Cano-Lozano y Buela-Casal, 2005; Pineda, Zapata y Donado, 2014), y es definida por la Organización Mundial de la Salud (OMS, 1996) como aquella percepción que cada individuo tiene de su lugar en la existencia, en el contexto cultural y sistema de valores en los que vive, así como la relación con sus objetivos, expectativas, normas e inquietudes. Por otro lado, las personas con sobrepeso y obesidad afrontan aquellos estigmas relacionados con la figura y el peso corporal, proveniente del cliché de que las personas con obesidad son diferentes en términos conductuales y de personalidad, considerándolos con poca fuerza de voluntad (León-Sánchez, Jiménez-Cruz, y Gonzalo, 2015) y poco atractivos, comentarios negativos que llegan a afectar la autoestima y, de igual forma, pueden conllevar a la adaptación de conductas alimentarias de riesgo, diversos estudios han encontrado una asociación entre la obesidad y una menor calidad de vida percibida, estos individuos con un índice de masa corporal (IMC) más alto, tienden a reportar puntuaciones bajas en las medias relacionadas con la calidad de vida (Salazar et al., 2016), se debe tomar en consideración la relación que ésta tiene con la esperanza de vida, así como el impacto de diversas enfermedades crónicas en los diversos dominios de cada uno de los individuos (Custorio, Murawski, Elizathe y Rutsztein, 2017).

Con respecto a lo anterior, tanto el sobrepeso como la obesidad están relacionadas con enfermedades que pueden ser tanto físicas como psicológicas, a pesar de las diversas investigaciones e intervenciones por parte del Estado, que advierten de las complicaciones que conllevan, aun así, existe una alta prevalencia de población mexicana con sobrepeso y obesidad. El propósito del presente trabajo fue comparar la calidad de vida relacionada con la salud (CVRS) entre personas clasificadas por su estado de nutrición (EN); normopeso, sobrepeso y obesidad, así como también por sexo.

\section{Método}

\section{Diseño}

El diseño de esta investigación fue de tipo no experimental y transversal, ya que sólo se realizó una medición de la calidad de vida relacionada con la salud, haciendo uso de la estadística descriptiva se explican sus características y propiedades presentes en la población de estudio, posteriormente se realizó una comparación de las medias estadísticas entre grupos divididos por su estado de nutrición y por sexo (Hernández, Fernández y Baptista, 2014). 


\section{Participantes}

La muestra fue de tipo no probabilística e intencional, conformada por 202 adultos (84 hombres y 118 mujeres) con una $M_{\text {edad }}=39.74$ años $(D E=13.77)$. Como criterio de inclusión se consideró que fueran derechohabientes del Instituto de Seguro Social del Estado de México y Municipios (ISSEMYM), mayores de edad que firmaran el consentimiento informado, que no presentaran alguna enfermedad de tipo crónico-degenerativa (independientemente de su estado de nutrición), y que hayan sido medidos y pesados para poder ser ubicados en alguno de los tres grupos correspondientes a su IMC, siendo: normopeso (59 sujetos), sobrepeso (78 sujetos) y obesidad (65 sujetos).

\section{Medidas}

Estado de nutrición. Calculado a través del índice de masa corporal (IMC), con la fórmula: peso (kg) dividido entre la estatura al cuadrado $\left(\mathrm{m}^{2}\right)$. Fue considerada la clasificación de la OMS (2017), como: normopeso (18.5-29.9), sobrepeso (25.0-29.9) y obesidad ( $\geq 30$ ).

Calidad de vida relacionada con la salud (CVRS): Se utilizó la versión en español del cuestionario 36Item Short Form Health Survey (SF-36) de Zúniga, Carrillo-Jiménez, Fos, Gandek y Medina-Moreno (1999), validado en población mexiquense por Arzate, Guadarrama, Veytia y Guzmán (manuscrito en preparación). Esta escala incluye 33 preguntas bajo un formato mixto (Frecuencia y Guttman), distribuidas en seis dimensiones pertenecientes a la CVRS, que explicaron $61.8 \%$ de la varianza total, siendo: Función física (FF), Salud mental (SM), Rol físico (RF), Vitalidad (VT), Salud general (SG) y Rol emocional (RE). El instrumento cuenta con una confiabilidad de alpha de Cronbach de .92 para el total del instrumento y, en cuanto a sus dimensiones va desde .79 a .91.

\section{Procedimiento}

La aplicación de los instrumentos se llevó dentro de las instalaciones de la Clínica Alfredo del Mazo del ISSEMYM, ubicada en la ciudad de Toluca, Edo. de México. Se acudió de manera personal con cada uno de los consultantes, a los cuales después de haber pasado a la toma de signos -peso y estatura- se les pidió su participación entregándoles el consentimiento informado, se resolvieron todas las dudas que estos externaron acerca de la investigación. A todos aquellos participantes que asintieron, firmando el consentimiento informado, se les aplicó el SF-36. Al terminar la aplicación, se procedió a la captura de datos en el programa SPSS V.19, para realizar los análisis estadísticos correspondientes, que fueron: descriptivos, $t$ de Student y ANOVA.

\section{Resultados}

De la muestra de 202 participantes se contó con 118 (58.4\%) mujeres y 84 (41.6\%) hombres. Del total de los participantes, $50(24.8 \%)$ indicaron ser solteros y 107 (53.0\%) casados. Con $M_{\text {edad }}=39.74$ años $(D E=13.77)$, con una $M_{\text {peso }}=73.48$ kilogramos $(D E=15.49)$ y la $M_{e s-}$ tatura fue de 1.62 metros $(D E=0.09)$, correspondiente a una $M_{I M C}=27.76 \mathrm{~kg} / \mathrm{mtr}^{2}(D E=4.77)$. La muestra fue distribuida según su IMC en: normopeso $(n=59)$, sobrepeso $(n=78)$ y obesidad $(n=65)$. Con respecto a la escolaridad de los participantes, 2 ( $1 \%$ ) indicaron no contar con escolaridad alguna, 43 (21.3\%) educación básica, 76 (37.6\%) educación media y 81 (40.1\%) educación superior (Tabla 1).

En la tabla 2 se pueden observar las puntuaciones promedio obtenidas por la muestra general en cada una de las dimensiones de CVRS, donde FF predominó con una puntuación considerada como muy buena; consecutivamente, las dimensiones RE, RF, VT y SM presentaron puntuaciones consideradas como buenas; por último, la puntuación en la dimensión SG (57.97) fue considerada regular.

En la tabla 3 se puede observar que el grupo con sobrepeso presentó mayor puntuación en las dimensiones SM, RF, VT y RE; no obstante, en el caso de las dimensiones FF y SG, fue el grupo normopeso el que presentó mayores puntuaciones. En el análisis de comparación realizado mediante la prueba ANOVA no se encontraron diferencias estadísticamente significativas en ninguna de las dimensiones de CVRS entre los tres grupos (normopeso, sobrepeso y obesidad).

En la tabla 4 se puede observar la comparación entre hombres y mujeres mediante el estadístico $t$ de Student, en donde el grupo de hombres presentó 
Tabla 1. Datos sociodemográficos.

\begin{tabular}{|c|c|c|c|c|c|c|c|c|c|c|c|}
\hline \multirow[t]{2}{*}{ Muestra } & \multirow[t]{2}{*}{ Edad } & \multirow[t]{2}{*}{ Peso } & \multirow[t]{2}{*}{ Estatura } & \multirow[t]{2}{*}{ IMC } & \multicolumn{3}{|c|}{ Estado de nutrición } & \multicolumn{4}{|c|}{ Escolaridad } \\
\hline & & & & & Normopeso & Sobrepeso & Obesidad & Ninguna & Básica & $\begin{array}{c}\text { Media } \\
\text { Superior }\end{array}$ & Superior \\
\hline $\begin{array}{l}\text { Total } \\
(202)\end{array}$ & $\begin{array}{c}39.74 \\
( \pm 13.77)\end{array}$ & $\begin{array}{l}73.48 \mathrm{~kg} \\
( \pm 15.49)\end{array}$ & $\begin{array}{c}1.62 \\
( \pm .09)\end{array}$ & $\begin{array}{c}27.76 \\
( \pm 4.77)\end{array}$ & $\begin{array}{c}59 \\
(29.2 \%)\end{array}$ & $78(38.6 \%)$ & $\begin{array}{c}65 \\
(32.2 \%)\end{array}$ & $\begin{array}{c}2 \\
(1 \%)\end{array}$ & $\begin{array}{c}43 \\
(21.3 \%)\end{array}$ & $\begin{array}{c}76 \\
(37.6 \%)\end{array}$ & $\begin{array}{c}81 \\
(40.1 \%)\end{array}$ \\
\hline $\begin{array}{c}\text { Hombres } \\
84 \\
(41.6 \%)\end{array}$ & $\begin{array}{c}41.2 \\
( \pm 14.9)\end{array}$ & $\begin{array}{l}82.8 \mathrm{~kg} \\
( \pm 15.6)\end{array}$ & $\begin{array}{c}1.7 \\
( \pm .06)\end{array}$ & $\begin{array}{c}28.7 \\
( \pm 4.7)\end{array}$ & $\begin{array}{c}17 \\
(20.2 \%)\end{array}$ & $35(41.7 \%)$ & $\begin{array}{c}32 \\
(38.1 \%)\end{array}$ & 0 & $\begin{array}{c}17 \\
(20.2 \%)\end{array}$ & $\begin{array}{c}34 \\
(40.5 \%)\end{array}$ & $33(39.3)$ \\
\hline $\begin{array}{c}\text { Mujeres } \\
118 \\
(58.4 \%)\end{array}$ & $\begin{array}{c}38.7 \\
( \pm 12.9)\end{array}$ & $\begin{array}{c}66.8 \\
( \pm 11.5)\end{array}$ & $\begin{array}{c}1.57 \\
( \pm .07)\end{array}$ & $\begin{array}{c}27.1 \\
( \pm 4.8)\end{array}$ & $\begin{array}{c}42 \\
(35.6 \%)\end{array}$ & $43(36.4 \%)$ & $\begin{array}{c}33 \\
(28 \%)\end{array}$ & $2(1.7 \%)$ & $\begin{array}{c}26 \\
(22 \%)\end{array}$ & $\begin{array}{c}42 \\
(35.6 \%)\end{array}$ & $\begin{array}{c}48 \\
(20.3 \%)\end{array}$ \\
\hline
\end{tabular}

Tabla 2. Estadísticos descriptivos por dimensiones de calidad de vida en la muestra general.

\begin{tabular}{lcccccc}
$\mathbf{N = 2 0 2}$ & Función física & Salud mental & Rol físico & Vitalidad & Salud general & Rol emocional \\
\hline$M$ & 81.49 & 69.22 & 75.42 & 70.94 & 57.97 & 77.56 \\
$D E$ & 22.86 & 19.79 & 29.69 & 19.37 & 19.682 & 35.38 \\
\hline
\end{tabular}

mayores puntuaciones en todas las dimensiones de calidad de vida; sin embargo, sólo en las dimensiones FF, SM, VT y RE se encontraron diferencias estadísticamente significativas a favor de los hombres.

En la tabla 5 se presenta la comparación entre los grupos por nivel educativo; Básico (o menor), Media y Superior. Al realizar el análisis de ANOVA se encontró diferencia estadísticamente significativa en la dimensión de FF, presentando una mayor puntuación los grupos de educación Media y Superior en comparación con los de nivel educativo Básico, asimismo, se encontró diferencia en la dimensión de SG entre los grupos con nivel Básico y Superior.

\section{Discusión}

En el presente estudio la muestra general presentó un IMC de $27.76 \mathrm{~kg} / \mathrm{m}^{2}$ el cual bajo la clasificación por estado de nutrición propuesto por la OMS (2017) se considera como sobrepeso, coincidiendo con los datos reportados por la ENSANUT (2012; 2016). Con respecto a la evaluación de la CVRS, la muestra total clasificó la dimensión de SG como regular, considerando que no es excelente como ellos quisieran, y que en el futuro existe cierta posibilidad de llegar a empeorar. Sin embargo, analizando las demás dimensiones, se puede observar que en éstas se presentan puntuaciones óptimas, puesto que no manifiestan problemas o limitaciones para realizar actividades cotidianas (FF) o laborales (RF). Por otro lado, en las puntuaciones de las dimensiones relacionadas con aspectos emocionales se puede apreciar que la SM fue percibida como buena, ya que a pesar de que llegaran a presentar problemas personales, estos no los limitan para realizar actividades relacionadas tanto con su trabajo (RE) como con sus relaciones interpersonales, manifestando sensación de energía y vitalidad (VT).

$\mathrm{Al}$ observar las puntuaciones de CVRS entre los grupos por su estado de nutrición, se puede apreciar que el grupo con sobrepeso presentó las puntuaciones más altas en la mayoría de las dimensiones, correspondientemente en SM, RF, VT y RE; seguido del grupo normopeso en FF y SG. No obstante, a pesar de que el grupo con obesidad no sobresalió en alguna de las dimensiones, las puntuaciones que obtuvo se encuentran en parámetros considerados desde regular a buena CVRS, contrario a lo que indica la literatura, 
Tabla 3. Comparación de las dimensiones de calidad de vida entre los estados de nutrición.

\begin{tabular}{|c|c|c|c|c|c|}
\hline $\begin{array}{l}\text { Estado de } \\
\text { nutrición }\end{array}$ & Dimensión & $M$ & $D E$ & $F$ & $p$ \\
\hline $\begin{array}{l}\text { Normopeso } \\
\text { (59) }\end{array}$ & \multirow{3}{*}{$\begin{array}{l}\text { Función } \\
\text { física }\end{array}$} & 85.78 & 20.43 & \multirow{3}{*}{2.83} & \multirow{3}{*}{.06} \\
\hline $\begin{array}{l}\text { Sobrepeso } \\
\text { (78) }\end{array}$ & & 82.55 & 21.22 & & \\
\hline $\begin{array}{l}\text { Obesidad } \\
(65)\end{array}$ & & 76.32 & 26.01 & & \\
\hline $\begin{array}{l}\text { Normopeso } \\
\text { (59) }\end{array}$ & \multirow{3}{*}{$\begin{array}{l}\text { Salud } \\
\text { mental }\end{array}$} & 66.04 & 16.49 & \multirow{3}{*}{1.34} & \multirow{3}{*}{.26} \\
\hline $\begin{array}{l}\text { Sobrepeso } \\
\text { (78) }\end{array}$ & & 71.62 & 19.28 & & \\
\hline $\begin{array}{l}\text { Obesidad } \\
(65)\end{array}$ & & 69.23 & 22.82 & & \\
\hline $\begin{array}{l}\text { Normopeso } \\
\text { (59) }\end{array}$ & \multirow{3}{*}{ Rol físico } & 77.16 & 28.50 & \multirow{3}{*}{0.99} & \multirow{3}{*}{.37} \\
\hline $\begin{array}{l}\text { Sobrepeso } \\
\text { (78) }\end{array}$ & & 77.65 & 27.84 & & \\
\hline $\begin{array}{l}\text { Obesidad } \\
(65)\end{array}$ & & 71.17 & 32.76 & & \\
\hline $\begin{array}{l}\text { Normopeso } \\
\text { (59) }\end{array}$ & \multirow{3}{*}{ Vitalidad } & 69.75 & 16.59 & \multirow{3}{*}{0.16} & \multirow{3}{*}{.85} \\
\hline $\begin{array}{l}\text { Sobrepeso } \\
\text { (78) }\end{array}$ & & 71.47 & 19.76 & & \\
\hline $\begin{array}{l}\text { Obesidad } \\
(65)\end{array}$ & & 71.38 & 21.39 & & \\
\hline $\begin{array}{l}\text { Normopeso } \\
\text { (59) }\end{array}$ & \multirow{3}{*}{$\begin{array}{l}\text { Salud } \\
\text { general }\end{array}$} & 60.85 & 17.45 & \multirow{3}{*}{1.47} & \multirow{3}{*}{.23} \\
\hline $\begin{array}{l}\text { Sobrepeso } \\
\text { (78) }\end{array}$ & & 58.40 & 18.44 & & \\
\hline $\begin{array}{l}\text { Obesidad } \\
(65)\end{array}$ & & 54.85 & 22.67 & & \\
\hline $\begin{array}{l}\text { Normopeso } \\
\text { (59) }\end{array}$ & \multirow{3}{*}{$\begin{array}{c}\text { Rol } \\
\text { emocional }\end{array}$} & 72.88 & 38.39 & \multirow{3}{*}{0.78} & \multirow{3}{*}{.46} \\
\hline $\begin{array}{l}\text { Sobrepeso } \\
\text { (78) }\end{array}$ & & 80.34 & 33.31 & & \\
\hline $\begin{array}{l}\text { Obesidad } \\
(65)\end{array}$ & & 78.46 & 35.07 & & \\
\hline
\end{tabular}

ya que la obesidad es considerada como una enfermedad de tipo crónico-degenerativo que influye en el desarrollo óptimo de la calidad de vida. También se puede apreciar que a pesar de ser el grupo que no
Tabla 4. Comparación de las dimensiones de calidad de vida por sexo.

\begin{tabular}{lccccc} 
Dimensión & Sexo & $\boldsymbol{M}$ & $\mathbf{D E}$ & $\boldsymbol{t}$ & $\boldsymbol{p}$ \\
\hline Función física & Hombre & 85.18 & 20.27 & & \\
& Mujer & 78.86 & 24.29 & 2.01 & .05 \\
Salud mental & Hombre & 73.61 & 17.75 & & \\
& Mujer & 66.10 & 20.64 & 2.70 & .008 \\
Rol físico & Hombre & 79.54 & 27.81 & & \\
& Mujer & 72.49 & 30.74 & 1.67 & .10 \\
Vitalidad & Hombre & 75.36 & 16.57 & & \\
& Mujer & 67.80 & 20.64 & 2.88 & .004 \\
Salud general & Hombre & 60.89 & 19.50 & & \\
& Mujer & 55.89 & 19.63 & 1.79 & .07 \\
Rol emocional & Hombre & 85.71 & 27.03 & 2.99 & .003 \\
& Mujer & 71.75 & 39.37 & & \\
\hline
\end{tabular}

sobresale en alguna de las dimensiones, en el caso de SM, VT y RE sus puntuaciones se encontraron por arriba del grupo con normopeso. Cabe mencionar que estas escalas tienen relación con el manejo de las emociones, así como con la sensación de energía y vitalidad. Dato que tiene relación con lo encontrado por Medrano-Vázquez et al. (2014), quienes refieren que el IMC tiene influencia en la disminución de la percepción del bienestar físico, pero no así con el bienestar emocional. A pesar de que algunos grupos presentaron puntuaciones más altas que otros en las diferentes dimensiones, al realizar la prueba de ANOVA no se encontraron diferencias estadísticamente significativas entre ellos.

En cuanto a los análisis por sexo se encontraron resultados similares a los de Medrano-Vázquez et al. (2014) y Salazar et al. (2016), en donde los hombres presentaron puntuaciones más elevadas en todas las dimensiones de CVRS en comparación con las mujeres, las cuales obtuvieron menor puntuación en áreas tanto físicas como mentales, no obstante, al realizar el análisis de $t$ Student sólo se encontraron diferencias estadísticamente significativas en las dimensiones de FF, SM, VT y RE. Cabe mencionar que las puntuaciones obtenidas por las mujeres se encuentran en un rango considerado como buena CVRS, salvo la dimensión SG (55.88), que se considera como regular. En el 
Tabla 5. Comparación de las dimensiones de calidad de vida con respecto al nivel educativo.

\begin{tabular}{|c|c|c|c|c|c|c|c|c|c|c|}
\hline \multirow{2}{*}{ Dimensiones } & \multirow{2}{*}{ Levene } & \multicolumn{2}{|c|}{$\begin{array}{c}\text { Nivel básico } \\
n=45\end{array}$} & \multicolumn{2}{|c|}{$\begin{array}{l}\text { Nivel medio } \\
\quad n=76\end{array}$} & \multicolumn{2}{|c|}{$\begin{array}{c}\text { Nivel superior } \\
\quad n=81\end{array}$} & \multirow[t]{2}{*}{$F$} & \multirow{2}{*}{$p$} & \multirow[t]{2}{*}{ Post-Hoc } \\
\hline & & $M$ & $D E$ & $M$ & $D E$ & $M$ & $D E$ & & & \\
\hline Función Física & .000 & 73.21 & 31.41 & 84.06 & 19.27 & 83.68 & 19.31 & 3.913 & .022 & $\begin{array}{c}\text { 1Vs } 2,3 \\
2 \text { vs } 1 \\
3 \text { vs } 1\end{array}$ \\
\hline Salud Mental & 0.063 & 67.04 & 23.00 & 68.60 & 21.02 & 71.03 & 16.52 & 0.647 & .525 & \\
\hline Rol Físico & 0.009 & 67.70 & 34.56 & 76.13 & 29.84 & 79.04 & 26.01 & 2.169 & .117 & \\
\hline Vitalidad & 0.404 & 73.44 & 21.13 & 70.20 & 20.17 & 70.25 & 17.64 & 0.481 & .619 & \\
\hline Salud Ceneral & 0.575 & 51.67 & 18.65 & 59.01 & 19.88 & 60.49 & 19.54 & 3.146 & .045 & $\begin{array}{l}1 \text { Vs } 3 \\
3 \text { Vs1 }\end{array}$ \\
\hline Rol Emocional & 0.091 & 69.63 & 38.81 & 78.07 & 36.33 & 81.48 & 32.06 & 1.646 & .195 & \\
\hline
\end{tabular}

caso de la comparación por medio de ANOVA entre los grupos por nivel educativo se presentaron diferencias significativas en las dimensiones de FF y SG, en la primera dimensión tanto el grupo con nivel educativo medio y superior, presentaron mayor puntuación en comparación con el grupo de nivel educativo básico (o menor), en la segunda dimensión solo se encontró diferencia entre los grupos con nivel básico y superior, estos fueron resultados similares a los ya reportados por Barajas, Robledo, Tomás, Sanz, García y Cerrada (1998), en otras palabras, tal y como lo indica De Vincezi y Tudesco (2009), la educación presenta una relación con la $\mathrm{CV}$, ya que tanto la educación formal como no formal, permite el desarrollo de habilidades que favorecen la búsqueda de recursos que permitan mejorar las condiciones de bienestar.

A pesar de que se encontraron diferencias estadísticamente significativas entre hombres y mujeres, así como por nivel educativo, no fue así entre los grupos por estado de nutrición (normopeso, sobrepeso y obesidad), ya que las puntuaciones obtenidas indican que poseen una percepción que va desde regular (solo en SG) a buena CVRS. Al parecer, esto indica que las personas con sobrepeso y obesidad no perciben que su condición sea un problema, pudiendo ellos favorecer la prevalencia de dichas condiciones (Rodriguez Barrera, Bastidas, Genta y Olaya-Contreras, 2016), tal y como lo ha venido reportando la ENSANUT (2012; 2016). Tanto el sobrepeso como obesidad son condiciones que desencadenan diversas enfermedades (e.g., cáncer, diabetes, hipertensión, etc.), y a pesar de la intervención del Estado, no se ha podido disminuir su prevalencia, por lo que se considera importante seguir realizando investigaciones que retomen otros tipos de variables, como pueden ser: psicológicas, económicas, culturales y demográficas, que ayuden a explicar desde otra perspectiva la influencia que tienen el sobrepeso y la obesidad.

Una de limitaciones del presente estudio fue el número de participantes, al considerar solo aquellos sujetos que no presentaran alguna enfermedad de tipo crónico-degenerativo ajeno a su EN la muestra se vio reducida, además es importante considerar otros métodos para la medir la grasa corporal como lo son: la medición de los pliegues subcutáneos, impedancia bioeléctrica, medición de la circunferencia de cintura, entre otros (Moreno, 2012). Con respecto a la metodología usada cabe recalcar que una de las principales limitaciones de los estudios transversales, si bien, nos permitió describir las características de nuestra, sin embargo, no determinan causalidad (Vallejo, 2002), por lo que es importante complementar tanto con otros diseños de investigación como con un equipo de trabajo multidisciplinario, que ayuden a corroborar los datos encontrados.

En conclusión, en el presente estudio no se encontraron diferencias estadísticamente significativas al comparar la CVRS entre los sujetos con normopeso, sobrepeso y obesidad, a diferencia de otros estudios, en los cuales indican que el aumento de IMC está 
relacionado con una disminución de la CVRS. No está de más mencionar, que en esta investigación se excluyeron a los sujetos que presentaran alguna enfermedad de tipo crónico-degenerativa (independiente a su EN), pudiendo ser esta una causa para no encontrar resultados similares a otras investigaciones, no obstante, los resultados analizados al realizar las comparaciones tanto por sexo como por nivel educativo indican que estas variables pueden estar asociadas con la percepción de cada individuo sobre su CVRS, de ahí que debe ser considerada desde un enfoque multifactorial e interdisciplinario, que permita crear estrategias relacionadas con la promoción de la salud, otorgando el desarrollo de habilidades y capacidades encaminadas a la modificación de las condiciones sociales, ambientales y económicas (Álvarez, 2013).

\section{Referencias}

Alcaraz, A. M., Ferrer, M. y Parrón, T. (2015). Calidad de vida en los pacientes obesos y su cambio tras cirugía bariátrica a medio y largo plazo. Nutrición Hospitalaria, 31(5), 2033-2046. https://doi.org/10.3305/nh.2015.31.5.8792

Álvarez, M. (2013). La promoción y prevención: pilares para la salud y la calidad de vida. Correo Científico Médico, 17(1), 79-82.

Arzate, G., Guadarrama, R., Veytia, M. y Guzmán, R. M. E. (2018). Validación del cuestionario SF-36 en población mexiquense. Manuscrito en preparación.

Barajas, M. A., Robledo, E., Tomás, N., Sanz, T., García, P. y Cerrada, I. (1998). Calidad de vida relacionada con la salud y obesidad en un centro de atención primaria. Revista Española de Salud Pública, 72(3), 221-231.

Barquera-Cervera, S., Campos-Nonato, I., Rojas, R. y Rivera, J. (2010). Obesidad en México: Epidemiología y políticas de salud para su control y prevención. Gaceta Médica de México, 146(6), 397-407.

Barquera, S., Campos-Nonato, I., Hernández-Barrera, L., Pedroza, A. y Rivera-Dommarco, J. A. (2013). Prevalencia de obesidad en adultos mexicanos 2000-2012. Salud Pública de México, 55(2), 151-160.

Barrera-Cruz, A., Molina-Ayala, M. A. y Rodríguez-González, A. (2013). Escenario actual de la obesidad en México. Revista Médica del Instituto Mexicano del Seguro Social, 51(3), 292-299.

Bertheau, E. L., Cruz-Quintana, F., Pappous, A. y Rio-Valle, J. S. (2016). Actitudes explícitas e implícitas hacia la obesidad en estudiantes de cultura física. Revista de Psicología del Deporte, 25(1), 91-96.

Custorio, J., Murawski, B., Elizathe, L., y Rutsztein, G. (2017). Calidad de vida relacionada con la salud: Análisis factorial exploratorio del RAND-26 en mujeres de Buenos Aires. Revista Argentina de Ciencias del Comportamiento, 9(2), 34-49.

De Vincezi, A. y Tudesco, F. (2009). La educación como proceso de mejoramiento de la calidad de vida de los individuos y de la comunidad. Revista Iberoamericana de Educación, 49(7), 1-12.

Dueñas, G. O., Del Carmen, L. M., Zamora G. I. y Saunas, G. (2005). ¿Qué es la calidad de vida para los estudiantes?. Revista de Neuro-Psiquiatría, 68(3-4), 212-221.

Encuesta Nacional de Salud y Nutrición. (2012). Encuesta Nacional de Salud y Nutrición 2012: Resultados nacionales. Cuernavaca, México: Instituto Nacional de Salud Pública. https://doi.org/10.1017/CBO9781107415324.004

Encuesta Nacional de Salud y Nutrición. (2016). Encuesta Nacional de Salud y Nutrición de Medio Camino 2016. Cuernavaca, México: Instituto Nacional de Salud Pública. https://doi.org/10.21149/8593

Esquivias-Zavala, H., Reséndiz-Barragán, A. M., García, F. y Elías-López, D. (2016). La salud mental en el paciente con obesidad en protocolo para cirugía bariátrica. Salud Mental, 39(3), 165-173. https://doi.org/10.17711/ SM.0185-3325.2016.015

Gálvez, A., Rosa, A. R., García-Cantó, E., Rodríguez, P. L., Pérez-Soto, J. J., Tarraga, L. y Tarraga, P. (2015). Estado nutricional y calidad de vida relacionada con la salud en escolares del sureste español. Nutrición Hospitalaria, 31(2), 737-743. https://doi.org/10.3305/nh.2015.31.2.8468

García-García, E., De la Llata-Romero, M., Kaufer-Horwitz, M., Tusié-Luna, M. T., Calzada-León, R., Vázquez-Velázquez, V., ...Sotelo-Morales, J. (2008). La obesidad y el síndrome metabólico como problema de salud pública: Una reflexión. Salud Pública de México, 50(6), 530-547. https://doi.org/10.1590/S0036-36342008000600015

Hernández, R., Fernández, C. y Baptista, P. (2014). Metodología de la investigación. México: Mc Graw-Hill.

Jiménez, S. M., Rodríguez, A. y Díaz, M. E. (2013). La obesidad en Cuba: Una mirada a su evolución en diferentes grupos poblacionales. Revista Cubana de Alimentación y Nutrición, 23(2), 12.

León-Sánchez, R., Jiménez-Cruz, B. E. y Gonzalo, J. A. (2015). Creencias de estudiantes de secundaria españoles y mexicanos sobre las causas de la obesidad. Acta de Investigación Psicológica, 5(2), 2062-2076.

López-Alarcón, M. G. y Rodríguez-Cruz, M. (2008). Epidemiología y genética del sobrepeso y la obesidad: 
Perspectiva de México en el contexto mundial. Boletín Médico del Hospital Infantil de México, 65(6), 421-430.

Medrano-Vázquez, M., Rojas-Russell, M. E., Serrano-Alvarado, K., Florez-Alarcón, L., Aedo-Santos, Á. y López-Cervantes, M. (2014). Asociación del índice de masa corporal y el autocontrol con la calidad de vida relacionada con la salud en personas con sobrepeso/ obesidad. Psychologia: Avances de la Disciplina, 8, 13-22.

Moreno, M. (2012). Definición y clasificación de la obesidad. Revista Médica Clínica Las Condes, 23(2), 124-128. https://doi.org/10.1016/S0716-8640(12)70288-2

Miró, E., Cano-Lozano, M. y Buela-Casal, G. (2005). Sueño y calidad de vida. Revista Colombiana de Psicología, 14, 11-27.

Organización Mundial de la Salud. (1996). ¿Qué calidad de vida? / Grupo de la OMS sobre la calidad de vida. Foro Mundial de la Salud, 17(4), 385-387. Recuperado de: https://apps.who.int/iris/handle/10665/55264

Organización Mundial de la Salud. (2017). Obesidady sobrepeso. Disponible en http://www.who.int/es/news-room/ fact-sheets/detail/obesity-and-overweight

Pineda, M. L., Zapata, M. y Donado, J. H. (2014). Calidad de vida relacionada con la salud de los estudiantes de pregrado de la Facultad de Medicina de la Universidad
Pontificia Bolivariana, 2013. Medicina U.P.B., 33(2), 117-123.

Rodríguez, J. C., Bastidas, M., Genta, G. y Olaya-Contreras, P. (2016). Calidad de vida percibida por los escolares con sobrepeso y obesidad, de sectores populares de Medellín, Colombia. Universitas Psychologica, 15(2), 301. https://doi.org/10.11144/Javeriana.upsy15-2.cves

Salazar, J. G., Torres, T. M., Aranda, C. y López, A. (2016). Calidad de vida relacionada con la salud y obesidad en el personal docente universitario de Jalisco, México. Actualización en Nutrición, 17(3), 2250-7183.

Unikel, C., Díaz de León, C. y Rivera, J. A. (2016). Conductas alimentarias de riesgo y correlatos psicosociales en estudiantes universitarios de primer ingreso con sobrepeso y obesidad. Salud Mental, 39(3), 141-148. https:// doi.org/10.17711/SM.0185-3325.2016.012

Vallejo, M. (2002). El diseño de investigación: una breve revisión metodológica. Archivos de Cardiología en México, 72(1), 08-12.

Zúniga, M. A., Carrillo-Jiménez, G. T., Fos, P. J., Gandek, B. y Medina-Moreno, M. R. (1999). Evaluación del estado de salud con la Encuesta SF-36: Resultados preliminares en México. Salud Pública de México, 41(2), 110-118. 